\title{
Planar and Cylindrical Active Microwave Temperature Imaging: Numerical Simulations
}

\author{
J. M. Rius, Student Member, IEEE, C. Pichot, L. Jofre, Member, IEEE, \\ J. C. Bolomey, N. Joachimowicz, A. Broquetas, Member, IEEE, and M. Ferrando, Member, IEEE
}

\begin{abstract}
$A b$ stract-Active microwave imaging systems for biomedical appli cations have been gaining attention in recent years. The need for iriternal structure information imposes the use of diffraction tomo graphy approaches. Two different geometries, planar and cylindrical, have been used for this purpose. In this paper we piresent a comparative study at $2.45 \mathrm{GHz}$ concerning both measurement and reconstruction parameters for the two configuration s. For the sake of comparison, a numerical model consisting of two nonconcentric cylinders is considered and reconstructed using both geometries from simulated experimental data. The scatter 'ed fields and reconstructed images allow to extract very useful information about dynamic range, sensitivity, resolution and cuantitative image accuracy for the choice of the configuration in a particular application.

The geometries chosen are planar and cylindrical array configurations. Both geometries can measure forward and backward scattered fields. The backscattering measurement improves the image resolution and reconstruction in lossy mediums, but, on the other hand, has several dynamic range difficulties. This tradeoff between only forward and forward-backward field measurement is analyzed using a planar array for forward scattering and a cylindrical one for forward-backward.

As differential temperature imaging is a weakly scattering problem, Born approximation algorithms can be used. The simplicity of Born reconstruction algorithms and the use of FFT make them very attractive for real-time biomedical imaging systems [19].
\end{abstract}

\section{INTRODUCTION}

D IFFERENT types of radiation are actually used for non invasive testing of biological bodies. Each of them has its own specific attributes, and the reconstructed image depends on the way in which the radiation interacts with matter [1].

The microwave region of the electromagnetic spectrum offers the access to an information which is related to the electric properties of the matter. These properties, usually expressed as

Manuscript received December 3, 1990; revised April 27, 1992. This work was supported by CAICYT (Spanish Committee for Scientific and Technical Research under Grant 1165-84), FISS (Spanish National Institute for Health under Grant 84/2112), Spanish-French Cooperation Program (Grant 30/135) and Spanish-British Cooperation Program (Grant 17/173). This work was also supported by the European Commission of the CEE in the frame of the Collaboration COMAC-BME Project "Optimization of Hyperthermia Technology and Assessment of Clinical Efficacy in Treatment of Cancer."

J. M. Rius, L. Jofre, A. Broquetas, and M. Ferrando are with E.T.S.E. Telecommunicació, Universitat Politècnica de Catalunya, Apdo. correos 30002, 08080 Barcelona, Spain.

C. Pichot, J.C. Bolomey, and N. Joachimowicz, are with Laboratoire des Signaux et Systemes (CNRS/ESE), Ecole Supérieure d'Electricité, Plateau de Moulon, F91192 Gif-sur-Yvette Cedex, Paris, France.

IEEE Log Number 9202263. a complex permittivity (permitivity and conductivity), translate in macroscopic terms the fine structure of the tissues at the molecular level, and therefore depend on its physical or physiological factors as well as the interrogation frequency [2].

The microwave propagation in biological tissues shows some specific properties.

- The attenuation is high, in particular for the tissues with a high water content as muscles, liver, kidney. However, soft tissues such as fat, lungs and bones present lower microwave absorption [3]. It is in those soft tissues where ultrasound energy suffers the greatest attenuation.

- At the same frequency, the wavelength in the tissues are reduced up to eight times compared to the air [4].

- Unlike X-ray imaging, where the wavelength is very small as compared to the magnitude of interesting object details, for microwaves, effects of wave propagation can not be explained in terms of rays, and diffraction effects have to be considered [5].

Active microwave imaging systems have not been developed for use in biosystems due to a number of apparent limitations related with the resolution and penetration. Although there was a first attempt at the beginning of the 70's [6], in fact they were the first experiments of Larsen and Jacobi [7], [8], at the end of the decade, that show the potentiality of the method. The basic principle employed consists of immersing the target in water and working in the lower region of the microwave spectrum (1-10 GHz.). The most significant points of the procedure are the following.

a) Water, being a medium with dielectric constant close to those of human body, improves the coupling and penetration of the electromagnetic radiation into the body.

b) The resolution of diffraction-limited imaging systems is determined by the wavelength of the interrogation radiation. When operating in water, with a relative permittivity close to 75 , there is a contraction in wavelength and therefore in resolution close to 9 .

c) The high attenuation of water at these frequencies avoids the multipath propagation external to the interrogated body.

Water is not the only possible matching medium. For instance, fat tissues could be better matched using lower permittivity liquids [9].

The choice the operating frequency is a tradeoff between resolution and depth of penetration. The requirement for penetration with deep-lying organs argues for a lower frequency 
of operation. In contrast, diffraction-limited imaging systems require high operating frequencies for enhanced resolution. The 1 to $6 \mathrm{GHz}$ region appear to be optimum for microwave imaging of the human body [10].

The first studies were devoted to projection imaging [7], [11], [12]. But, rapidly, tomographic reconstruction processes were considered. A first approach [13], close to X-rays CAT, failed for strongly inhomogeneous media. However, the diffraction effects have to be taken into account [14], [15] in order to obtain good quality images.

The use of microwave radiation in imaging biological structures has been gaining attention recently. The interest rely on its low cost and low complexity technology, as well as in its capacity to discriminate different tissues (permitivities ranges from 5 to 50 , compared to the few percent of densities in soft tissues) and to detect physiological changes, as solute concentration, perfusion, blood flow rate and temperature [16]-[19]. Moreover, the photon energy of microwaves is sufficiently low to prevent any ionization to occur in biological tissue at ordinary intensity levels.

The illumination and measurement geometry depend on the particular application and on the technology available. In this paper, by using a computer model, we compare the most significant parameters for the two basic configurations that have mainly been used in active microwave imaging: the planar geometry [18] and the cylindrical geometry [20]. The model consists of two nonconcentric cylinders with different permittivity values, simulating a general biological body.

Section II outlines the basics of the formulation for the two geometries. An effort has been made trying to enhance the common steps for both solutions.

The third section is devoted to compare the different measurement and reconstruction parameters for the two geometries on the basis of a common simulated structure.

\section{TOMOGRAPHIC FORMULATION}

Imaging techniques consist of illuminating the object with an incident radiation, measuring the diffracted fields, and obtaining an image of the distribution of the properties of the object interacting with the illuminating energy. Two kinds of microwave tomographic systems will be formulated, differing on the geometry of incident wave and the measurement antenna.

Tomography with diffracting energy, such as microwaves, must be modelled with a second-order linear differential equation, the inhomogeneous wave equation, which describes the electromagnetic field distribution in the object to be imaged. Since computing the object image from the scattered field measurements is an extremely difficult problem to solve, a first-order approximation has to be used, so that only weakly scattering objects can be reconstructed with reasonable error.

When incident radiation is concentrated on a slice of the object, and with object slowly varying in the third dimension, a bidimensional model can be used: dielectric objects and electromagnetic fields will be assumed to have properties varying only over the $x-y$ plane cross section. Using vertically polarized antennas in the $z$-axis direction, all electric field and current vectors will be $z$-directed, and accordingly we can use scalar field equations. The two dimensional model is valid only if the object is actually uniform in the $z$-direction inside the slice in which illuminating energy is concentrated. The reconstructed object will be the $(x, y)$ image of this slice.

\section{A. Spectral Formulation of Diffraction Problems}

The physical properties of the object which interact with microwave radiation are the electrical permittivity and con. ductivity. Since diffraction theory is based on coherent fiel $\mathrm{ds}$, phasor formulation of fields and currents will be used witt.1 the time dependence $e^{j \omega t}$.

Complex permittivity and wavenumber are then defined as follows:

- complex dielectric permittivity of the object under test: $\epsilon(\vec{r})$;

- complex dielectric permittivity of external medium: $\epsilon_{0}(\vec{r})$;

- complex wavenumber in the external medium: $K_{0}=$ $\omega \sqrt{\mu_{0} \epsilon_{0}}$, where $\mu_{0}$ is the vacuum magnetic permeability.

Let $E(\vec{r})$ represent the total electric field and $E^{i}(\vec{r})$ the incident field, that is to say, the field in the absence of the object. The scattered field is defined as

$$
E^{S}(\vec{r})=E(\vec{r})-E^{i}(\vec{r})
$$

and can be considered as the field generated by the equivalent electric current radiating in the external medium:

$$
E^{s}(\vec{r})=\iint_{S}-j w \mu_{0} J\left(\overrightarrow{r^{\prime}}\right) G\left(\vec{r}-\overrightarrow{r^{\prime}}\right) d \overrightarrow{r^{\prime}}
$$

where $G\left(\vec{r}-\overrightarrow{r^{\prime}}\right)$ is the Green's funcion, and

$$
J\left(\overrightarrow{r^{\prime}}\right)=-j w\left(\epsilon\left(\overrightarrow{r^{\prime}}\right)-\epsilon_{0}\right) E\left(\overrightarrow{r^{\prime}}\right)
$$

the equivalent induced electric current.

The bidimensional Fourier transform of the :quivalent current is defined through the equation

$$
\tilde{J}(\vec{K})=F T[J(\vec{r})]=\iint_{S} J(\vec{r}) e^{j \vec{K} \cdot \vec{r}} d \vec{r} .
$$

If the object complex permittivity is close to that of the surrounding medium, the scattered field is negligible in front of the incident one (weak scattering) and the Born approximation can be applied:

$$
\epsilon(\vec{r}) \sim \epsilon_{0} \rightarrow E(\vec{r}) \sim E^{i}(\vec{r}) .
$$

When the object is illuminated by a plane wave incident at an angle $\theta_{0}$, using a unit vector $\hat{\theta}_{0}$ in this direction, we have

$$
E^{i}(\vec{r})=e^{-j K_{0} \hat{\theta}_{c} \vec{r}}
$$

Equation (4), evaluated at $|\vec{K}|=K_{0}$, can be expressed as:

$$
\tilde{J}(\vec{K})=-j w \epsilon_{0} \tilde{C}\left(\vec{K}-K_{0} \hat{\theta}_{0}\right) \quad|\vec{K}|=K_{0}
$$




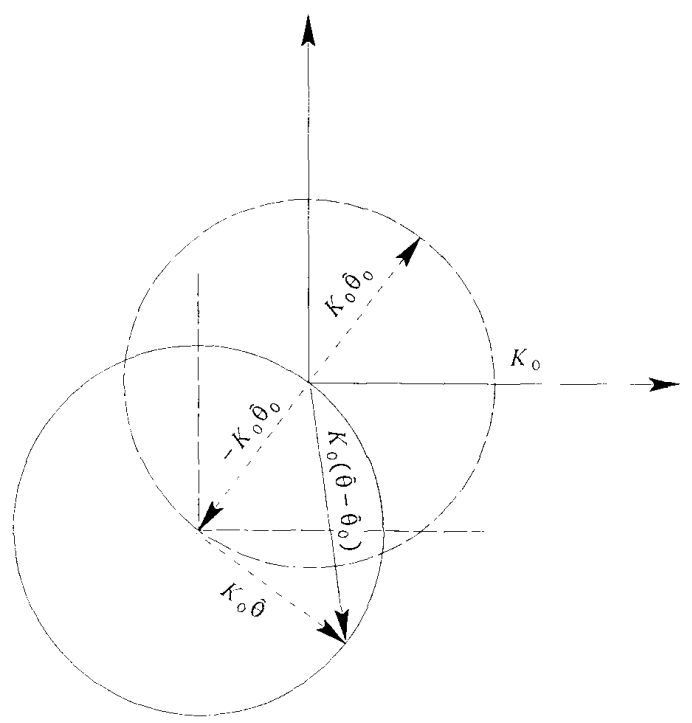

Fig. 1. Fourier domain for an incident plane wave. Under Born approximation, the bidimensional Fourier transform of the contrast profile is obtained in the Fourier domain over a circumference shifted on the direction opposite to the incident plane wave.

where

$$
\begin{aligned}
\tilde{C}\left(\vec{K}-K_{0} \hat{\theta}_{0}\right) & =F T[C(\vec{r})] \\
& =\iint_{S} C(\vec{r}) e^{j\left(\vec{K}-K_{0} \dot{\theta}_{0}\right) \cdot \vec{r}} d \vec{r}|\vec{K}|=K_{0}
\end{aligned}
$$

is the bidimensional Fourier transform of the contrast profile defined as

$$
C(\vec{r})=1-\frac{\epsilon(\vec{r})}{\epsilon_{0}}
$$

evaluated over circumferences of radius $K_{0}$ and centre $-K_{0} \hat{\theta}_{0}$ in the Fourier space (Fig. 1).

When the surrounding medium is lossy, the wavenumber $K_{0}$ is complex, and the Fourier transform becomes a Laplace one. However, in order to use the FFT algorithm the complex wavenumber must be approximated by its real part. This introduces an attenuation of the higher spatial frequencies, which produces "high-frequency" noise in the reconstructed image. For that reason, some low-pass filtering of the reconstructed image is necessary when backscattering information is processed, because it is associated to the higher spatial frequencies in the object spectrum.

As the bidimensional Fourier transform of the equivalent current evaluated at $|\vec{K}|=K_{0}$ can be easily obtained from scattered field measurements, the contrast profile of a weakly scattering object will be reconstructed through (7).

The reconstruction (7) gives information of the 2-D Fourier transform of the contrast profile only over a circumference shifted in direction opposite to the propagation of the incident field. Thus, different directions of incidence must be considered in order to obtain a bidimensional sampling inside a circle in the spectral domain (Fig. 2). Accordingly, the reconstructed

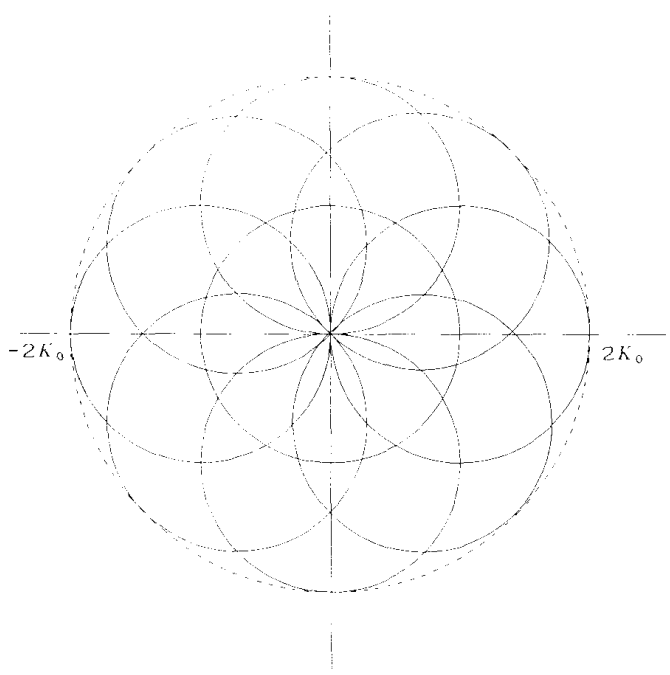

Fig. 2. Multiview Fourier domain. If multiple views are taken for different directions of illumination, the bidimensional Fourier transform of the contrast profile can be obtained inside a circle of radius $2 \kappa_{0}$ centered at the origin of the Fourier domain.
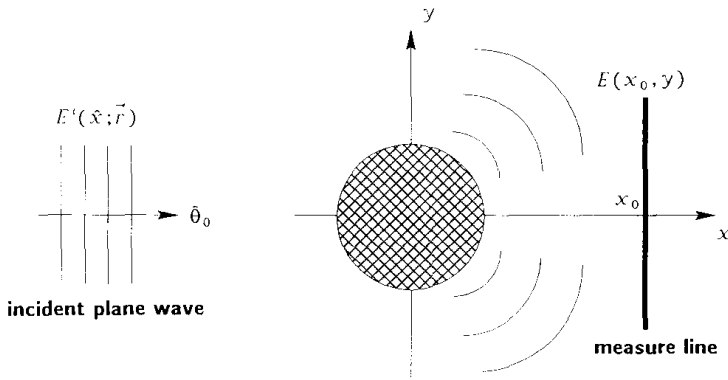

Fig. 3. Planar system measurement geometry (transmisison configuration) On planar geometry systems, the object is illuminated by a plane wave and the scattered field is measured along a probing line parallel to the incident wavefront. If another probing line is located at $-x_{0}^{\prime}$, both forward and backscattered fields can be measurcd.

contrast profile obtained through inversion of $\tilde{C}(\vec{K})$ will be a low-pass filtered version of the original one.

\section{B. Planar Geometry}

A tomographic system with planar geometry uses a plane wave incident field $E^{i}\left(\hat{\theta}_{0} ; \vec{r}^{\prime}\right)$ propagating on direction $\theta_{0}$, for simplicity $\theta_{0}=0^{\circ}$, and the total field $E\left(x_{o}, y\right)$ is measured along the line $x=x_{0}$ parallel to the incident wavefront (Fig. 3). Mechanical rotation of the object around $z$-axis provides different directions of incidence relative to the object.

The Fourier transform of the equivalent current can be obtained from the measured total field through the Fourier transform of (2) along $y$-direction. After some manipulation, see [18], the following expression can be obtained:

$$
\begin{gathered}
\tilde{E}^{s}\left(x_{0} . K_{y}\right)=-j w \mu_{0} \tilde{G}\left(\sqrt{K_{0}^{2}-K_{y}^{2}}\right) \cdot \tilde{J}\left(\sqrt{K_{0}^{2}-K_{y}^{2}}, K_{y}\right) \\
|\vec{K}|=K_{0}
\end{gathered}
$$


where $\tilde{E}^{s}\left(K_{y}\right)$ and $\tilde{G}\left(\sqrt{K_{0}^{2}-K_{y}^{2}}\right)$ are the angular spectrum of the scattered field and the Green's function, respectively,

$$
\begin{gathered}
\tilde{E}^{s}\left(x_{0}, K_{y}\right)=\int_{-\infty}^{\infty} E\left(x_{0}, y\right) e^{j k_{y} y} d y \\
\frac{e^{-j \sqrt{K_{0}^{2}-K_{y}^{2}} x_{0}}}{2 j \sqrt{K_{0}^{2}-K_{y}^{2}}}=\tilde{G}\left(\sqrt{K_{0}^{2}-K_{y}^{2}}\right)=\tilde{G}\left(K_{x}\right)=\frac{e^{-j K_{x} x_{0}}}{2 j K_{x}}
\end{gathered}
$$

being $K_{x}$ and $K_{y}$ the cartesian components of the real approximation to the propagation vector related to each of the plane wave components of the angular spectrum

$$
\begin{aligned}
\vec{K} & =\left(K_{x}, K_{y}\right) \quad K_{x}=K_{0} \cos \theta \\
|\vec{K}|^{2} & =K_{0}^{2}=K_{x}^{2}+K_{y}^{2} \quad K_{y}=K_{0} \sin \theta .
\end{aligned}
$$

Under Born approximation, the 2-D Fourier transform of the contrast profile is obtained through (7), (10), and (11):

$$
\tilde{C}\left(K_{0}\left(\hat{\theta}-\hat{\theta}_{0}\right)\right)=\frac{2 j K_{x}}{K_{0}^{2}} \tilde{E}^{s}\left(K_{y}\right) e^{j K_{x} x_{0}} .
$$

When the planar tomographic system measures only the forward scattered field through the object, only the components of the angular spectrum of the scattered field propagating towards positive $x\left(K_{x}>0\right)$ arrive at the measure line. These spectral components are associated to the right half of the circle $\left(K_{x}>0\right)$ in the spectral domain (Fig. 4). Thus, rotation of the object provides the 2-D Fourier transform of the contrast profile inside of circle of radius $\sqrt{2} K_{0}$ (Fig. 5) [18], [19]. If another probing line is located at $-x_{0}$ (Fig. 3), both forward and backscattered fields can be measured. In this case, which is not analyzed in this paper, rotation of the object provides 2-D Fourier transform of the contrast profile inside a circle of radius $2 K_{0}$ as indicated in Fig. 2 . The simplicity of the reconstruction algorithm and the use of FFT make it very attractive for real-time imaging systems [19].

\section{Cylindrical Geometry}

In cylindrical tomographic systems the measurement is performed not by a straight line, but by a circular array of isotropic antennas (Fig. 6). Different directions of illumination can be achieved by transmitting with each one of the omnidirectional elements, so that mechanical rotation of the object is avoided.

Although the incident field is not a plane wave, but a cylindrical one, and the measure antenna is a circular array, the spectral formulation developed for planar geometry imaging systems can be applied if a synthetic aperture approach is made.

As the circular array can measure, in principle, both the forward and backward scattered field, the Fourier transform of the contrast profile is obtained over the entire shifted circle in the spectral domain, and better resolution is achieved with forward-backscattering algorithms due to a less rigorous lowpass filtering than with forward scattering systems. However, in practice, backscattered fields are difficult to measure near

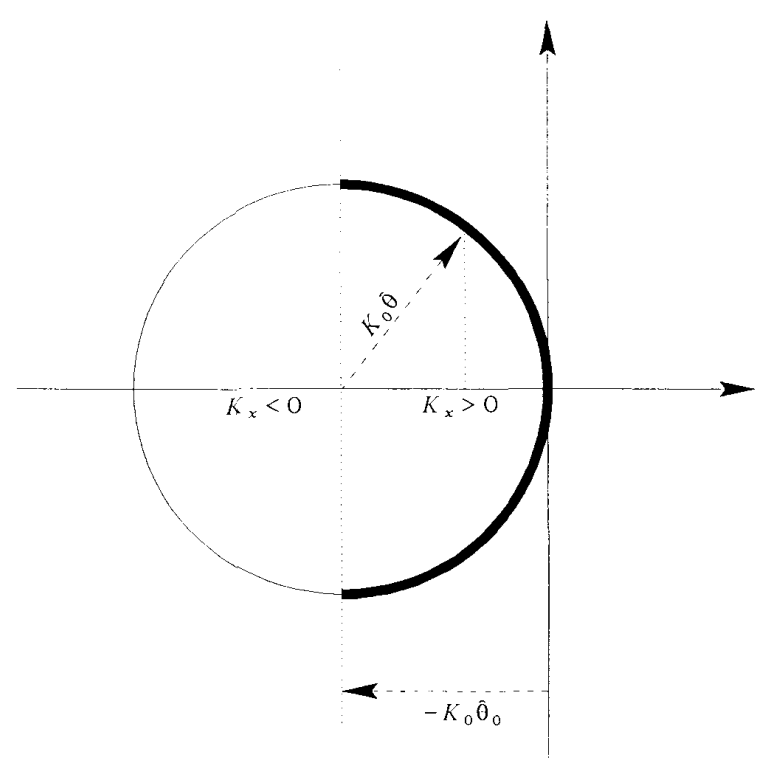

Fig. 4. Fourier domain for 1 view planar system. If only forward scattered field is measured on planar geometry systems, the bidimensional Fourier transform of the contrast profile is obtained only over half of the shifted circumferences. If backscattered field is also measured, the whole circumference is obtained.

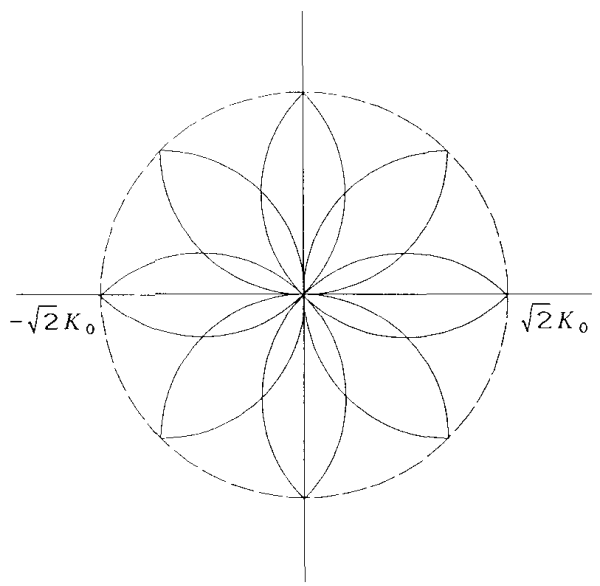

Fig. 5. Fourier domain for 8 view transmission planar system. When multiple views are taken on a transmission planar geometry system, the bidimensional Fourier transform of the contrast profic is obtained inside a circle of radius $\sqrt{2} K_{0}$ instead of $2 \kappa_{0}$.

the transmitting element, and resolution is not as good as theoretically expected.

Using a reciprocity theorem formulation, the bidimensional Fourier transform of the equivalent current can be easily obtained from the scattered field measured along an arbitrarily shaped cylindrical antenna, providing that plane waves in any direction can be synthesized by currents on the receiving antenna, acting as transmitter. The reciprocity theorem states that

$$
\int_{v^{\prime} a} \vec{J}_{a} \cdot \vec{E}_{b} d v_{a}=\int_{v_{b}} \vec{J}_{b} \cdot \vec{E}_{a} d v_{b}
$$




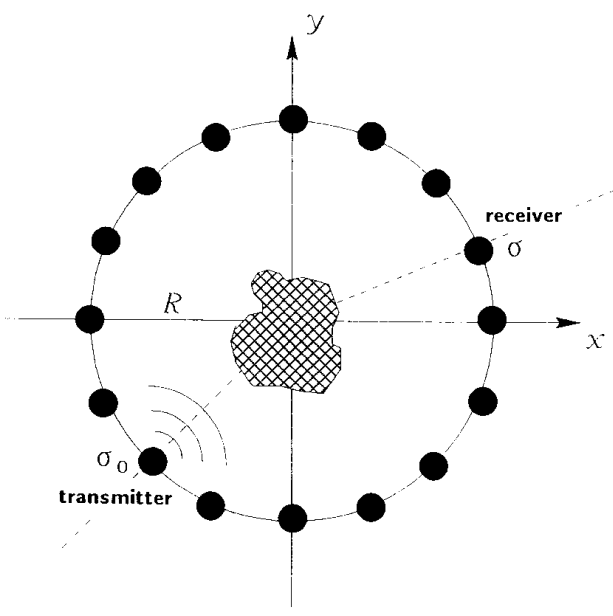

Fig. 6. Cylindrical geometry system. For cylindrical geometry systems, the measurement is achieved with a circular array of omnidirectional antennas. Different directions of illumination can be achieved by transmitting with each one of the omnidirectional elements.

where $\vec{J}_{a}$ and $\vec{J}_{b}$ are the electric currents existing in volumes $v_{a}$ and $v_{b}$, which radiate electric fields $\vec{E}_{a}$ and $\vec{E}_{b}$, respectively.

If we assume that $\overrightarrow{J_{b}}$ is the electric equivalent current in the object, induced by the incident field $\vec{E}^{i}\left(\vec{r} ; \hat{\theta}_{0}\right)$, and $\vec{J}_{a}$ is the electric current on the antenna acting as transmitter that radiates a plane wave field propagating along the unit vector $\hat{\theta}$, then we have that $\vec{J}_{b}$ radiates the scattered field $\vec{E}^{s}(\vec{r})$, which is measured along the antenna, and that $\vec{J}_{a}$ radiates the field $e^{-j K_{0} \hat{\theta} \cdot \vec{r}}$.

From (3) and (9) we get

$$
J_{b}(\vec{r})=\frac{1}{j w \mu_{0}} K_{0}^{2} C(\vec{r}) E\left(\vec{r} ; \hat{\theta}_{0}\right)
$$

substituting $J_{b}$ in (13), and considering (7) and (8), we obtain under Born approximation

$$
\tilde{C}\left(K_{0}\left(\hat{\theta}-\hat{\theta}_{0}\right)\right)=j w \mu_{0} \int_{v_{a}} J_{a}(\vec{r}, \theta) E^{s}\left(\vec{r} ; \hat{\theta}_{0}\right) d v_{a} .
$$

For a circular antenna of radius $R$, centered in the $x-y$ plane, (15) can be expressed as

$$
\tilde{C}\left(K_{0}\left(\hat{\theta}-\hat{\theta}_{0}\right)\right)=j w \mu_{0} \int_{0}^{2 \pi} I(\theta-\sigma) E^{s}\left(\sigma ; \hat{\theta}_{0}\right) R d \sigma
$$

where $I(\theta-\sigma)$ is the amplitude of the current distribution at angular position $\sigma$ along the antenna, that, when acting as transmitter, radiates inside the source ring a plane wave propagating at an angle $\theta$ in the direction $\hat{\theta}$

$$
I(\theta-\sigma)=\frac{4 j}{-j w \mu_{0} R} \frac{1}{2 \pi} \sum_{n=-\infty}^{\infty} \frac{j^{-n}}{H_{n}^{(2)}\left(K_{0} R\right)} e^{j n(\theta-\sigma)} .
$$

When the incident field is a cylindrical wave, we can expand the plane wave $E^{i}\left(\vec{r} ; \theta_{0}\right)$ as a superposition of cylindrical waves $E^{i}\left(\vec{r} ; \sigma_{0}\right)$ generated by line sources $I\left(\theta_{0}-\sigma_{0}\right)$ located as positions $\sigma_{0}$ along the circular antenna. Then it follows that

$$
E^{i}\left(\vec{r} ; \hat{\theta}_{0}\right)=\int_{0}^{2 \pi} I\left(\theta_{0}-\sigma_{0}\right) E^{i}\left(\vec{r} ; \sigma_{0}\right) R d \sigma_{0}
$$

and therefore the field scattered from an incident plane wave, measured along the circular antenna can be expressed as

$$
E^{s}\left(\sigma ; \hat{\theta}_{0}\right)=\int_{0}^{2 \pi} I\left(\theta_{0}-\sigma_{0}\right) E^{s}\left(\sigma ; \sigma_{0}\right) R d \sigma_{0}
$$

where $E^{s}\left(\sigma ; \sigma_{0}\right)$ is the scattered field measured at position $\sigma$, generated by an incident cylindrical wave produced by a unit source located at position $\sigma_{0}$ (Fig. 5). Finally, from (16) and (19) we obtain

$$
\begin{aligned}
\tilde{C}\left(K_{0}\left(\hat{\theta}-\hat{\theta}_{0}\right)\right)= & j w \mu_{0} R^{2} \int_{0}^{2 \pi} \int_{0}^{2 \pi} E^{s}\left(\sigma ; \sigma_{0}\right) \\
& \cdot I(\theta-\sigma) I\left(\theta_{0}-\sigma_{0}\right) d \sigma d \sigma_{0} .
\end{aligned}
$$

Equation (20) has the following physical interpretation: $E^{s}\left(\sigma ; \sigma_{0}\right)$ is convolved with the current that, existing along a circular array, generates a plane wave. This convolution transforms the circular geometry into a cartesian one, for both transmitter and receiver variables $\sigma$ and $\sigma_{0}$. As with planar configuration algorithm, this equation can be easily implemented by means of Fast Fourier Transforms (FFT), which allows real-time reconstructions [19], [20]. Further information about the algorithm theory and implementation can be found in [21].

\section{NumERICAL RESUlTS}

This section compares the relative performance of the forward and forward-backscattering measurement using, respectively, planar and cylindrical geometry reconstruction algorithms. The scattered fields have been computed in both cases by moment method, solving the linear system of equations by conjugate-gradient iterative algorithm.

The software used to compute scattered fields, for both geometries, and to reconstruct the object image for planar geometry have been developed at Laboratorie des Signaux et Systèmes, Ecole Supérieure d'Electricité, Gif-sur-Yvette, while the cylindrical geometry reconstruction program has been developed at AMR group, Department of Signal Theory and Communications, Polytechnic University of Catalonia, Barcelona.

Postprocessing algorithms have been applied to improve image quality when necessary.

- Gerchberg-Papoulis algorithm has been applied to planar geometry reconstructions when the number of views is small

- Cylindrical geometry reconstructions must be low-pass filtered in order to remove high-frequency noise due to backscattered field measurements.

\section{A. Numerical Model}

One of the possible applications of microwave tomography concerns medical imaging. As human body is composed mainly of water, and the complex dielectric permittivity of 


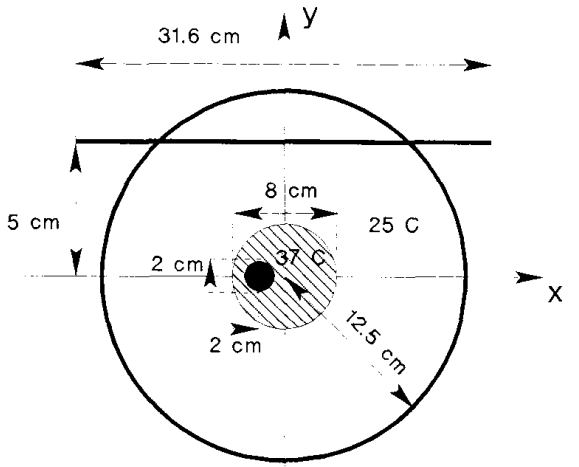

Fig. 7. Simulated model of biological object. For computer simulation purpose, a very rough biological object is modeled by a circular region of water of radius $4 \mathrm{~cm}$ at $37^{\circ} \mathrm{C}$ immersed in water at $25^{\circ} \mathrm{C}$. A local temperature change inside the body is simulated by a smaller, nonconcentric cylinder of water of radius $1 \mathrm{~cm}$ at $38^{\circ} \mathrm{C}$ or $41^{\circ} \mathrm{C}$

TABLE I

Complex permittivity and contrast profile of water

\begin{tabular}{cc}
\hline Complex permittivity & Contrast profilc \\
\hline$\epsilon\left(25^{\circ}\right)=77.27-j 9.29$ & $\left|C\left(25^{\circ}\right)\right|=0$ \\
$\epsilon\left(37^{\circ}\right)=73.49-j 0.55$ & $\left|C\left(37^{\circ}\right)\right|=0.0591$ \\
$\epsilon\left(38^{\circ}\right)=73.13-j 0.35$ & $\left|C\left(38^{\circ}\right)\right|=0.0646$ \\
$\epsilon\left(41^{\circ}\right)=72.34-j 5.88$ & $\left|C\left(41^{\circ}\right)\right|=0.076 .5$
\end{tabular}

water varies linearly with temperature, temperature variations inside human body can be detected and localized. This suggests an application to hyperthermia monitoring and tumor detection.

A complete set of computer simulations has been performed to test the capability of both algorithms to detect localized temperature variations inside water. A very rough biological object is modelled by a circular region of water at $37^{\circ} \mathrm{C}$ immersed in water at $25^{\circ} \mathrm{C}$. A local temperature change inside the body is simulated by a smaller, nonconcentric, cylinder of water at $38^{\circ} \mathrm{C}$ or $41^{\circ} \mathrm{C}$ (Fig. 7). We have used an operating frequency of $2.45 \mathrm{GHz}$ [19], [20], with a wavelength of $1.39 \mathrm{~cm}$ in water at $25^{\circ} \mathrm{C}$, which is a good tradeoff between attenuation and resolution.

1) Model Parameters: Our model is a weakly scattering object, because its contrast profile is low and its dimension not too large compared to the wavelength. Thus, total field is similar to incident field, and Born approximation can be applied. The complex permittivity and contrast profile of water at $2.45 \mathrm{GHz}$ for different temperatures are listed in Table I. In this range, the permittivity sensitivity with temperature is almost linear, equal to

$$
\frac{\Delta \epsilon^{\prime} / \epsilon_{0}^{\prime}}{\Delta T}=-3.9876 \cdot 10^{-3 \circ} C^{-1}
$$

2) Planar Geometry Simulation Parameters:

Length of measurement line:

$31.6 \mathrm{~cm}$

Number of receiving antennas:

67

Number of views:

Spacing between receiving antennas:
SCATTERED FIELD
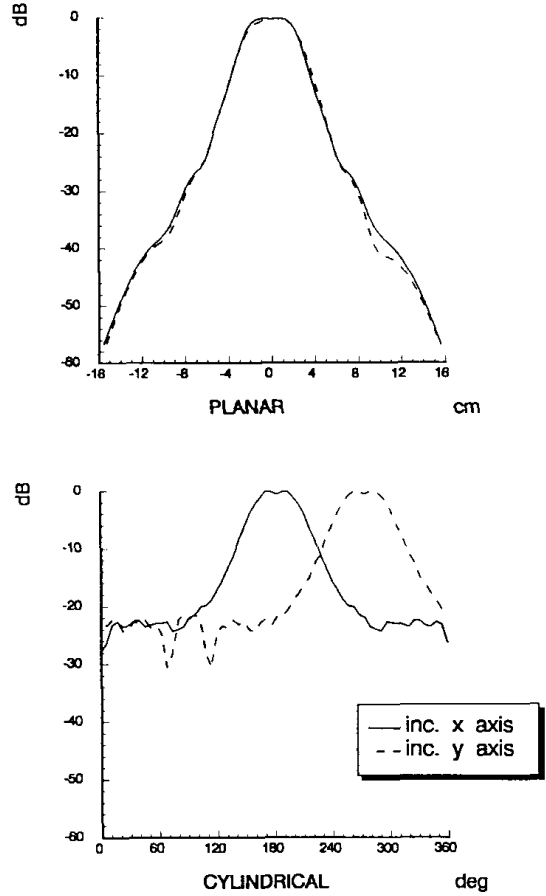

Fig. 8. Scattered field. Magnitude of the probing scattered field in $\mathrm{dB}$ for both planar and cylindrical geometry for local temperature change inside the cylinder of $4^{\circ}$. Two different directions of incidence have been considered, along $x$-axis $\left(0^{\circ}\right)$ and $y$-axis $\left(90^{\circ}\right)$. A minimum dynamic range of $30 \mathrm{~dB}$ for the cylindrical case and $45 \mathrm{~dB}$ for the planar one is needed to detect the difference between the two views.

Distance to the center of the object: $\quad 5 \mathrm{~cm}$ Dimension of reconstructed image matrix: $67 \times 67$

3) Cylindrical Geometry Simulation Parameters:

Number of transmitting antennas: $\quad 64$

Number of receiving antennas: $\quad 64$

Radius of the circular array: $\quad 12.5 \mathrm{~cm}$

Spacing between receiving antennas: $\quad 5.625^{\circ}=$

$1.23 \lambda$

Dimension of reconstructed image matrix: $64 \times 64$

\section{B. Scattered Field}

For comparison purpose the incident field at the center of the object is fixed: $0.5 \mathrm{~V} / \mathrm{m}$ in amplitude, zero degree in phase. Fig. 8 shows the magnitude of the probing scattered fields for both geometries. Two different views of the object have been plotted for different directions of incidence along the $x$-axis $\left(0^{\circ}\right)$ and $y$-axis $\left(90^{\circ}\right)$.

For the planar case the two curves overlap, because the probing line is always in the same relative position to the incident wave, while for the cylindrical case the two plotted curves are similar in shape, although shifted $90^{\circ}$.

Fig. 9 shows the magnitude of the differential fields, i.e. the difference between the scattered fields for a temperature change of $4^{\circ} \mathrm{C}$ and the scattered fields for no temperature change. 

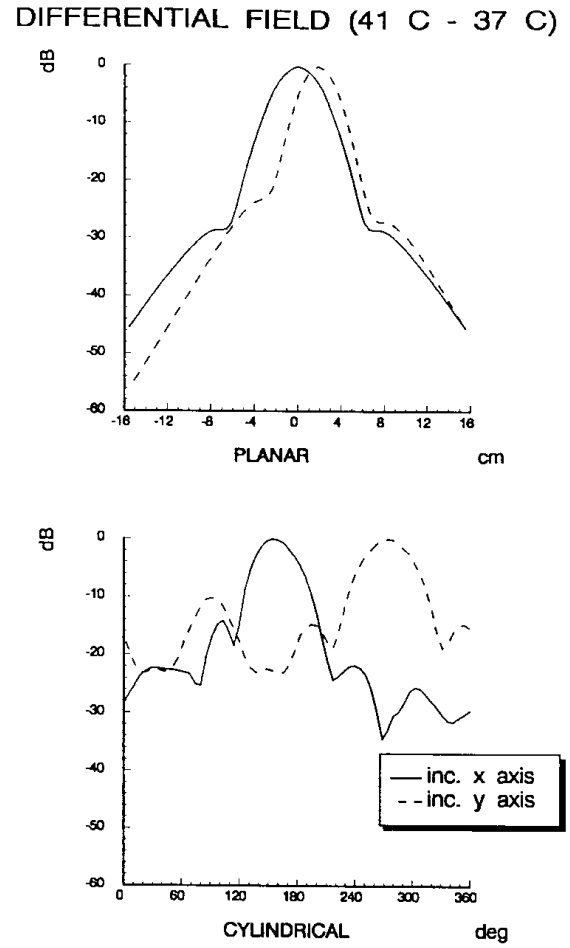

Fig. 9. Differential scattered field $\left(41^{\circ} \mathrm{C}-37^{\circ} \mathrm{C}\right)$. Magnitude of the difference between the scattered field measured for a $4^{\circ} \mathrm{C}$ temperature change and the scattered fields for no temperature change. Two views along $x$ and $y$ axis are shown. A minimum dynamic range of $35 \mathrm{~dB}$ is needed for the cylindrical case, and $60 \mathrm{~dB}$ for the planar one. The latter can be reduced down to $40 \mathrm{~dB}$ with a shorter probing line.

For practical purposes, only the probing field corresponding to a certain dynamic range, i.e., $30 \mathrm{~dB}$, has a clear influence on the reconstructed image. Because of the different behavior of the two measured patterns, the useful angular extend of the measured field for the cylindrical geometry will be larger than the equivalent one for the planar case.

This can be explained as follows.

The differential field can be considered as the field scattered by the temperature change. For the planar case, the temperature change location is close to the center of the measure line and far away from the ends. The maximum difference of distances is about $13 \mathrm{~cm}$, leading to an attenuation difference of about $30 \mathrm{~dB}$ [Fig. 10(a)].

In the cylindrical case, the temperature change location is near the center of the circular array, and therefore the distances to each one of the receiving elements are similar. The greatest distance difference is about $4 \mathrm{~cm}$, and the attenuation difference is about $9 \mathrm{~dB}$ [Fig. 10(b)].

On the other hand, the larger number of elements in the cylindrical array which measure fields within the specified dynamic range, provide a larger amount of information to be processed, which results in a slightly better spectral padding and image quality, at the price of a longer probing line.

\section{Reconstructed Images}

1) Absolute Reconstructions: Fig. 11 shows the recon-

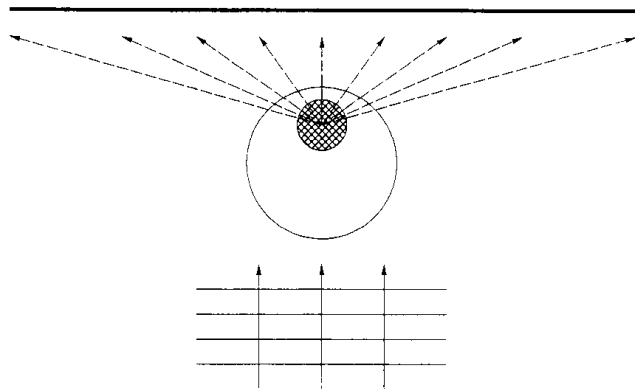

(a)

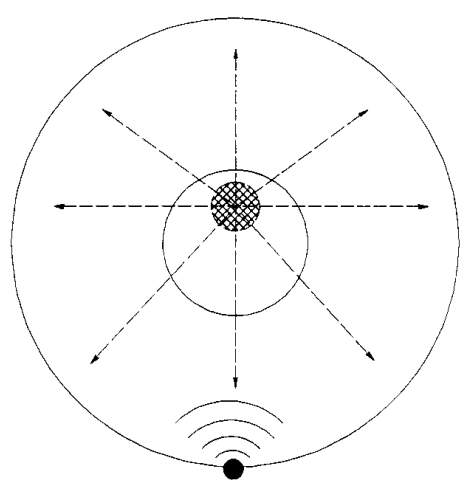

(b)

Fig. 10. Scattered field attenuation. The distance from the temperature change location to each one of the receiving elements of the measure array is approximately constant in the cylindrical geometry system [Fig. 10(b), but not in the planar one [Fig. 10(a)]. Thus, the scattered field arriving at the elements close to the ends of the measure line is strongly attenuated compared to the field at the center of the array. For that reason, the planar geometry system requires a dynamic range of about $20 \mathrm{~dB}$ greater than the cylindrical geometry one.

structed contrast profiles for temperature changes of 0,1 , and $4{ }^{\circ} \mathrm{C}$. Planar geometry reconstruction and cylindrical one are very similar. It must be noticed that cylindrical configuration has a larger spectrum coverage due to the back-scattered field measurement: circle of radius $2 K_{0}$ while only $\sqrt{2} K_{0}$ for the planar one. Accordingly, in theory the spatial resolution of an ideal backscattering system is better by a $\sqrt{2}$ factor.

However, images obtained by the cylindrical configuration from forward and backward scattering field measurements are not so better [see Fig. 11] for the following reasons.

- The backscattered field at elements very close to the transmitting one cannot be measured, and is approximated by zero. As backscattered fields correspond to high frequencies in the Fourier domain of the reconstructed object (Figs. 1 and 2), some spatial resolution is lost.

- When the FFT algorithm is used to compute the 2-D inverse Fourier transform of the object spectrum $\tilde{C}(\vec{K})$, the wavenumber $\vec{K}$ is assumed to be real valued. If the medium is lossy, some error is introduced in the higher frequencies of the object spectrum. As the backward field measurements are associated with this higher spatial frequencies, a low-pass image filter must be applied and some resolution is lost again. This filtering is not necessary when only forward scattering measurement is performed. 
CONTRAST PROFILE
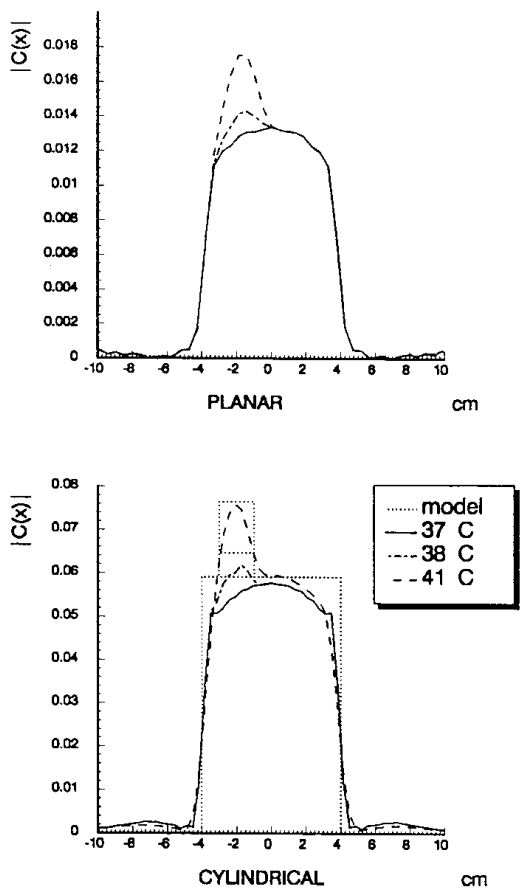

(a)

\section{CONTRAST PROFILE}
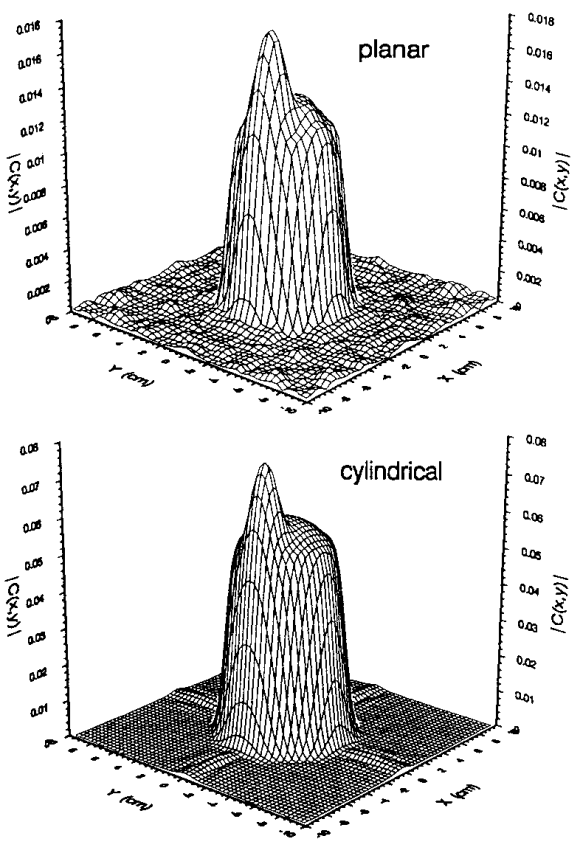

(b)

Fig. 11. Reconstruction of contrast profile for tempetature changes of $0^{\circ} \mathrm{C}$, $1^{\circ} \mathrm{C}$, and $4^{\circ} \mathrm{C}$. Planar geometry and cylindrical geometry reconstructions are very similar - although the cylindrical geometry should be in principle better by a factor $\sqrt{2}$ due to the backscattered field measurement.
DIELECTRIC PERMITTIVITY
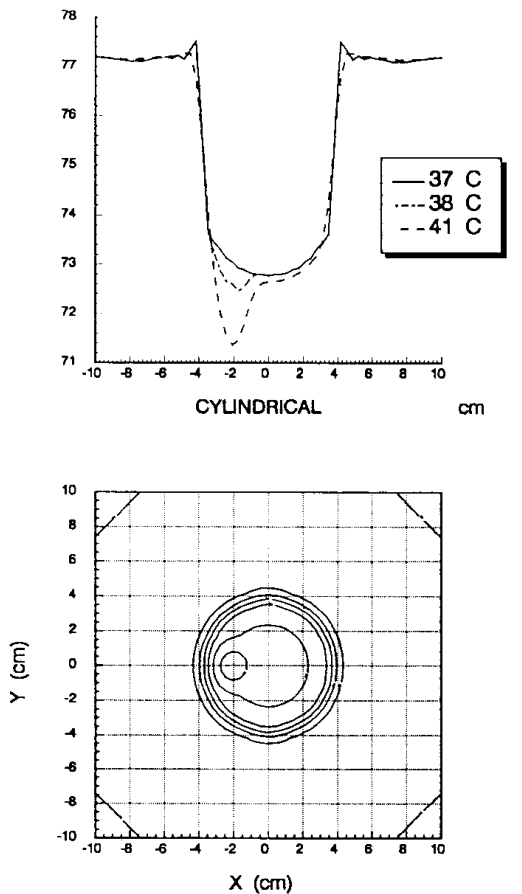

Fig. 12. Reconstruction of dielectric permittivity on cylindrical system.

As a conclusion, the image resolution obtained with the forward scattering planar configuration and the cylindrical one are very similar, although the larger spectrum coverage due to the backscattering measurement in the cylindrical case.

Cylindrical geometry reconstruction is performed taking into account the lossy medium with complex $K_{0}$ in (20), so that the correct value of the object spectrum $\tilde{C}$ is obtained and quantitative values of the contrast profile are obtained with very good approximation. The complex permittivity of the image can be easily derived from (9) (see Fig. 12), and a temperature map image can be obtained from the approximately linear relationship between contrast profile and temperature (Fig. 13).

The results of Fig. 11 have been obtained by the planar algorithm using $K_{x}$ real valued in (12). Thus, the highly attenuated amplitude of the scattered field due to the lossy medium is supposed to be caused by a less diffracting object and the reconstructed value of the contrast profile is lower than the original. Therefore, the planar geometry algorithm with $K_{x}$ real in (12) provides only a qualitative image of the contrast profile (Fig. 11).

2) Loss Correction in Planar Geometry Algorithm: When a quantitative reconstruction in a lossy medium is desired, a loss correction must be performed in planar geometry algorithm. This is done by taking $K_{x}$ complex valued in (12). With this correction, each component of the angular spectrum of the measured scattered field is amplified by a exponential factor equal to the attenuation in the lossy medium. This may be a major problem in an experimental system, because the noise related to signals arriving at both ends of the array is amplified 
TEMPERATURE MAP
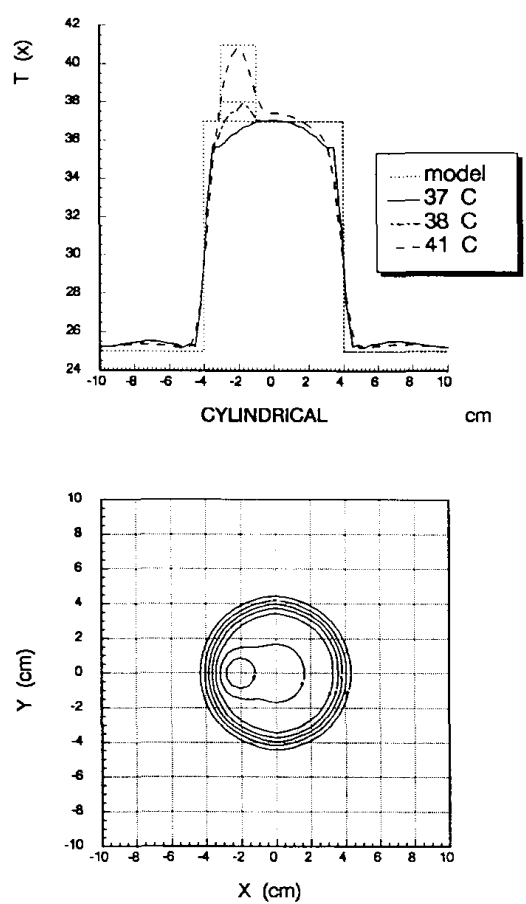

Fig. 13. Reconstruction of temperature map on cylindrical system. As the dielectric permittivity of water varies lineary with temperature, temperature maps obtained with the cylindrical system.

by the loss correction exponential factor. In general, the loss correction is very sensitive to the amplification of noise for the higher spatial frequencies.

Figs. 14 and 15 show the reconstructed images with both the planar and cylindrical geometry algorithms, for a temperature change of $1^{\circ} \mathrm{C}$, located at $x=0 \mathrm{~cm}$. A loss correction has been performed for the planar case, while for the cylindrical one the loss correction is implicit taking complex $K_{0}$ in (20).

Dielectric permittivity is reconstructed properly with both algorithms, but the planar geometry image has been distorted by losses correction, as shown in Fig. 14. A temperature map for both geometries is also shown in Fig. 15.

The main reason for the better results achieved with the cylindrical algorithm compared to the planar one with loss correction is that in lossless media the forward scattered field is much larger than the backscattered, but in lossy media the relative importance of the backscattered field increases with the loss tangent, so that the cylindrical algorithm takes advantage over the planar one. This point has been validated by numerical simulation: if in the cylindrical algorithm only the forward scattering information is used, the results are very similar to those of the planar with losses correction.

As a conclusion, it can be noticed in Figs. 14 and 15 that the backscattered field measurement in lossy media improves the image quality when a quantitative reconstruction is obtained.

3) Differential Reconstructions: An image of a localized temperature change in the object can be obtained subtracting the image of the object from the image of the object with the

\section{COMPLEX PERMITTIVITY}
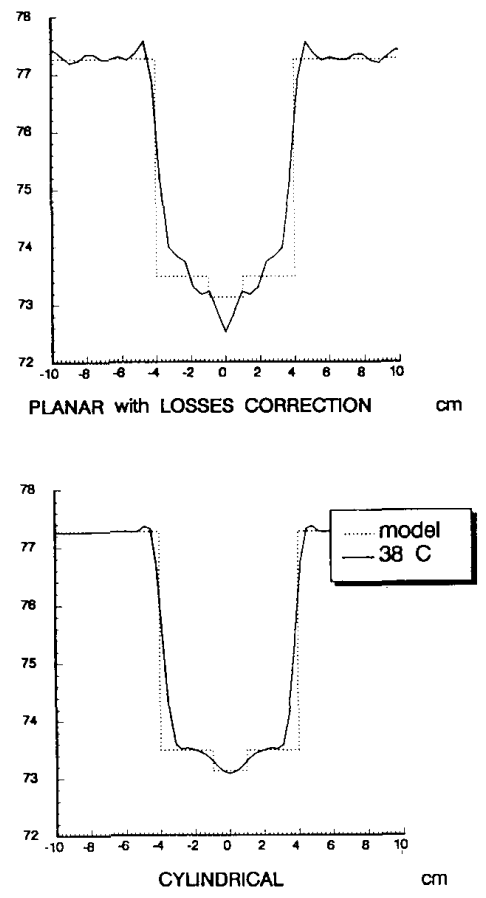

(a)

\section{CONTRAST PROFILE}
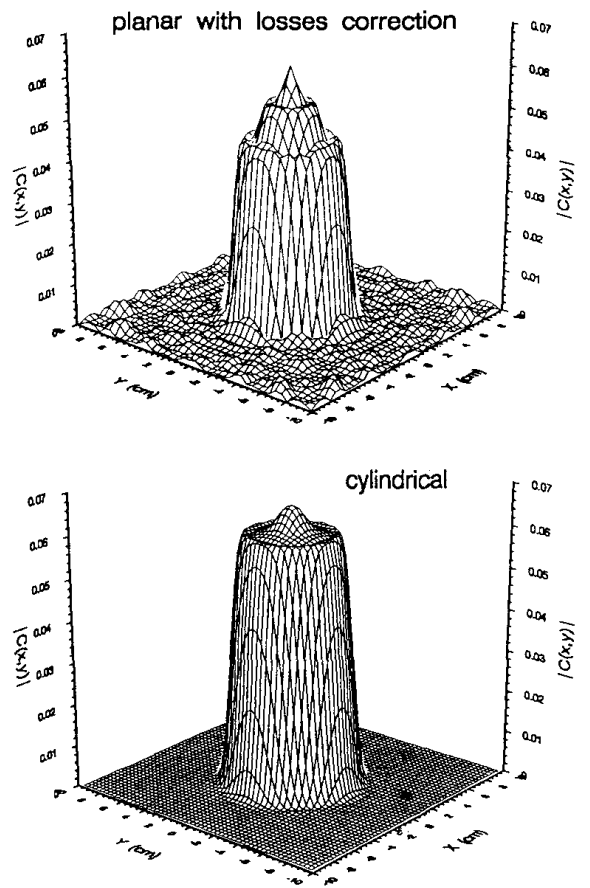

(b)

Fig. 14. Reconstruction of dielectric permittivity with losses correction. Dielectric permittivity is reconstructed properly with both algorithms but planar geometry image has been distorted by loss correction. 
TEMPERATURE MAP
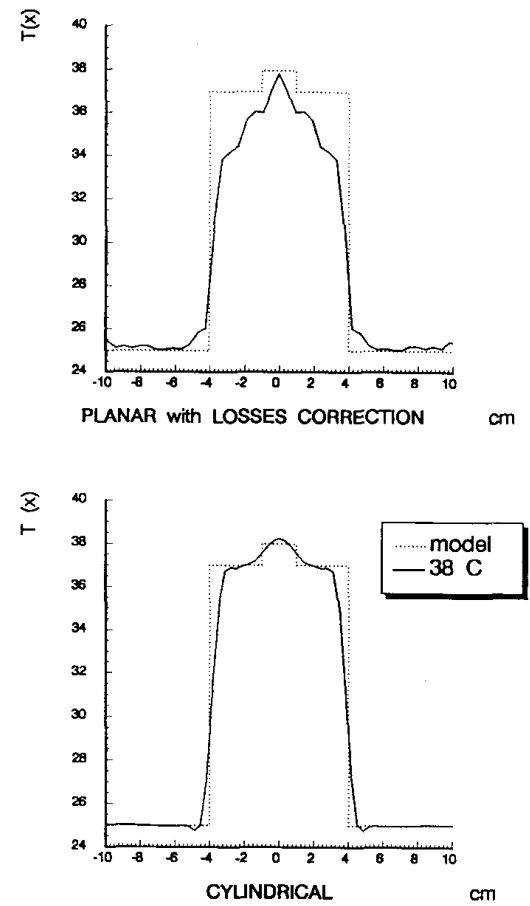

Fig. 15. Reconstruction of temperature map with losses correction. Absolute temperature maps for the cylindrical and planar geometry algorithms obtained from the permittivity presented in Fig. 14. Loss correction have been performed in the planar configuration.

permittivity variation. This technique appears to be very useful for hyperthermia monitoring, as removes the image of the object, leaving only the reconstruction of the contrast profile change. As permittivity varies linearly with temperature, a temperature map image can be easily obtained.

In differential reconstructions, Born approximation is less restrictive, because part of the error caused by the difference between the total field and the incident one is cancelled by subtraction. It can be easily shown [22] that the reconstructed differential contrast profile is

$\Delta C(\vec{r})=\frac{E_{2}(\vec{r})}{E^{i}(\vec{r})}\left(C_{2}(\vec{r})-C_{1}(\vec{r})\right)+\frac{E_{2}(\vec{r})-E_{1}(\vec{r})}{E_{i}(\vec{r})} C_{1}(\vec{r})$

where $C_{1}(\vec{r})$ and $C_{2}(\vec{r})$ are respectively, the contrast profile before and after the temperature change, and $E_{1}(\vec{r})$ and $E_{2}(\vec{r})$ the fields scattered by $C_{1}(\vec{r})$ and $C_{2}(\vec{r})$, respectively.

Small contrast profile variations can be detected in highcontrast objects because $E_{1}(\vec{r})$ is similar to $E_{2}(\vec{r})$. If the object does not fulfill Born approximation, but the contrast profile variation at $\vec{r}=\vec{r}_{0}$ does, then $E_{2}(\vec{r}) / E_{i}(\vec{r})$ is constant within the permittivity change region in the proximities of $r_{0}$, and the differential contrast profile reconstruction is proportional to the original contrast profile increment

$$
\Delta C(\vec{r})=\frac{E_{2}(\vec{r})}{E_{i}(\vec{r})}\left(C_{2}(\vec{r})-C_{1}(\vec{r})\right) .
$$

\section{DIFFERENTIAL CONTRAST PROFILE}
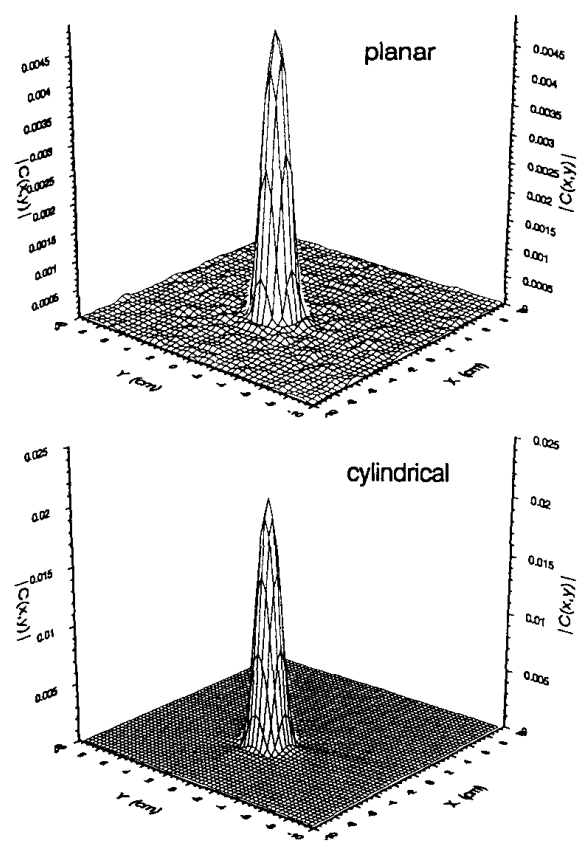

Fig. 16. Differential reconstruction of contrast profile without losses correction. Diefferential contrast profile images for both the planar and cylindrical systems.

The reconstructed differential contrast profile is shown in Fig. 16 for both geometries. As no loss correction is performed in Fig. 16, the planar geometry algorithm is not able to reconstruct properly the expected value $C\left(41^{\circ}\right)-C\left(37^{\circ}\right)=$ 0.017 . Fig. 17 shows a differential temperature map for the cylindrical case.

\section{Spectral-Processing}

1) Gerchberg-Papoulis Algorithm: When the number of view taken is not sufficient, the reconstructed image is distorted. The minimum number of views necessary to reconstruct properly the object is equal to the number of coefficients of the cylindrical-mode expansion of the scattered field (the proof is trivial from reciprocity theorem). This is approximately $N_{\phi}=2 K R$ where $K$ is the wavenumber and $R$ the radius of the minor circle containing the object.

For our simulated model, we have $2 K R=36$, so the 64 views reconstruction is good. When $N_{\phi}<2 K R$, the cylindrical coordinate Fourier-Bessel series of the object is undersampled in the angular coordinate, and we obtain an image with "directional spatial aliasing," as shown in Fig. 18 for a 4 views reconstruction.

This results can be improved adding a priori information. Gerchberg-Papoulis iterative algorithm has been applied to 4 view reconstructions of a centered $1^{\circ} \mathrm{C}$ temperature increment. Fig. 18 shows the improvement for 1 to 10 iterations. For a 
DIFFERENTIAL TEMPERATURE
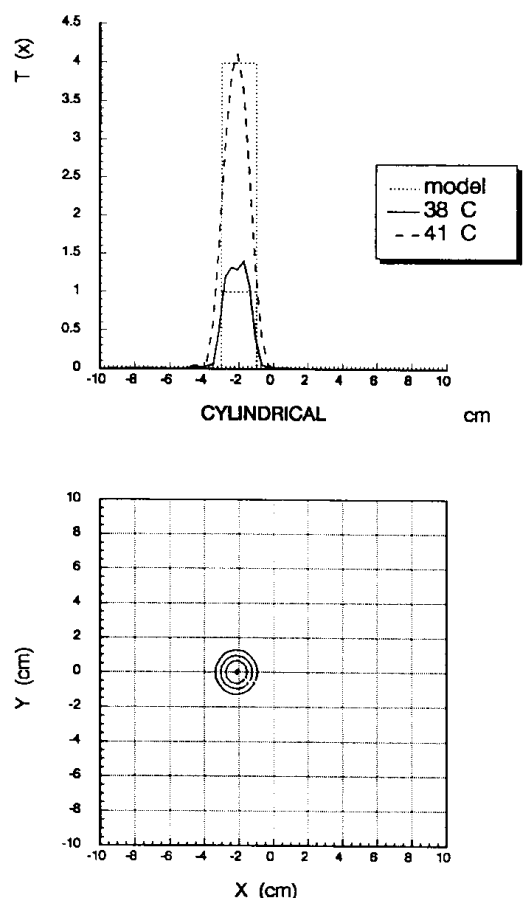

Fig. 17. Differential temperature map. A differential temperature map obtained for the cylindrical case.

larger number of iterations, the algorithm diverges slowly due to errors in the reconstruction procedure [23].

2) Low-Pass Image Filtering: The cylindrical geometry algorithm measures both the forward and backward scattered fields. In lossless media and for weakly scattering objects, the backscattered field measured near the transmitting antenna is much lower than the forward scattered field. In lossy media, the forward scattered field suffers greater attenuation than the backscattered. This relative increment of backscattered field is corrected in part by the reconstruction algorithm in lossy media.

As backscattered field information is related to highfrequency components of the reconstructed object, the image contains some high-frequency noise, which can be easily removed by a low-pass filter. We have tested cosinus filters with transfer function

$$
H(K)=A+(1-A)\left(1+\cos \left(\pi \frac{K}{K^{\prime}}\right)\right)^{n} \prod\left(\frac{K}{K^{\prime}}\right) .
$$

Optimum results have been obtained with $A=0, K^{\prime}=$ $1.8 K_{0}, n=0.55$ and $A=0, K^{\prime}=1.5 K_{0}, n=1.5$. Fig. 19 shows the transfer function of the low-pass filter and the Fourier transform of the image, before and after filtering. Fig. 20 shows the originally reconstructed image and the lowpass filtered one.

All the simulation results shown in this paper have been filters with transfer function (23), and the following parameter values:

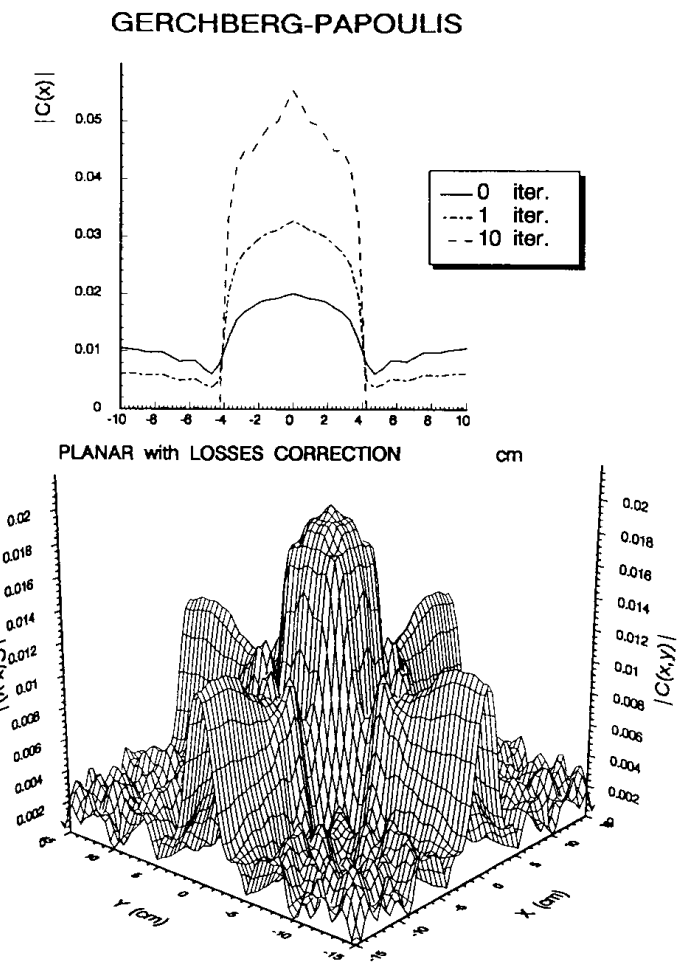

(a)
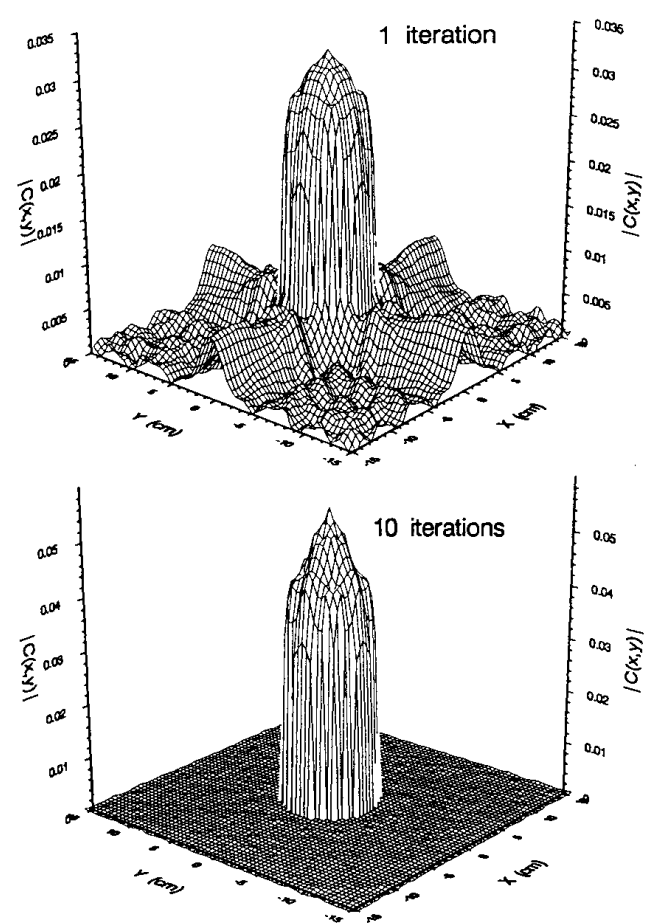

(b)

Fig. 18. Gerchberg-Papoulis iteration. When the number of views taken is less than $N_{0}=2 K^{-} R$ (about 36 in our example), the object cannot be reconstructed properly. The results can be improved adding a priori information by Gerchberg-Papoulis iterative algorithm. The picture shows that a 4 view reconstruction is considerably improved with 1 and 10 iterations. 
LOW-PASS IMAGE FILTER
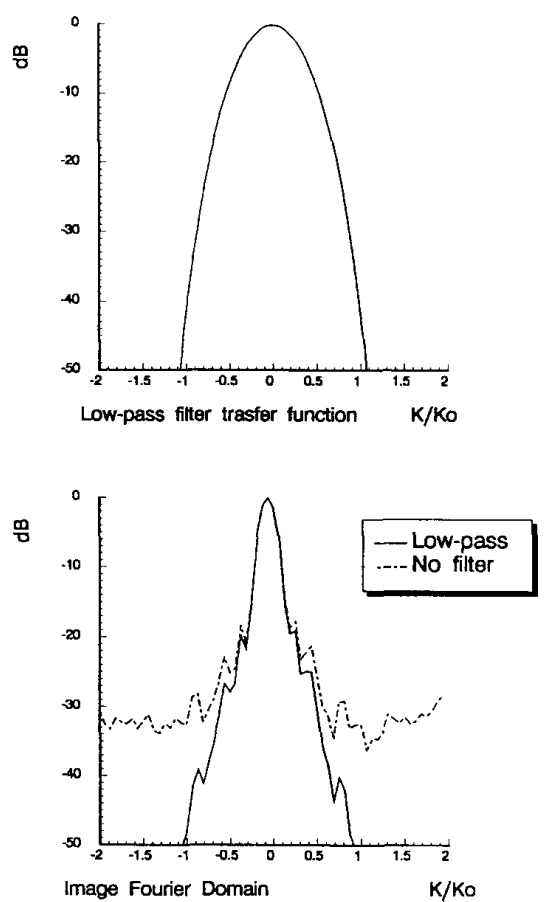

Fig. 19. Low-pass anti-aliasing image filtering on cylindrical systems. Cylindrical geometry system images contain high-frequency noise due to attenuation in lossy mediums. Thus, reconstructed images must be low-pass filtered Optimum results are obtained with the filter shown in Fig. 19.

- Temperature change at $x=-2 \mathrm{~cm}$ :

$$
\begin{array}{lll}
37^{\circ} \mathrm{C}: A=0, & K^{\prime}=1.8 K_{0}, & n=0.55 \\
38^{\circ} \mathrm{C}: A=0, & K^{\prime}=1.8 K_{0}, & n=0.55 \\
41^{\circ} \mathrm{C}: A=0, & K^{\prime}=1.5 K_{0}, & n=1.5
\end{array}
$$

- Temperature change at $x=0 \mathrm{~cm}$ :

$$
38^{\circ} \mathrm{C}: A=0, \quad K^{\prime}=1.5 K_{0}, \quad n=1.5
$$

Notice that the former provides sharper corners and faster rising times, while the latter a smoother image.

\section{CONCLUSIONS}

Both algorithms are able to perform accurate reconstructions of low contrast objects. Also, small changes in high contrast objects can be detected and localized, which has an important application in biomedical imaging [19], [20].

There are, though, some differences in the image quality and experimental limitations for the planar and the cylindrical geometries.

Although planar geometry systems could, in principle, measure the backscattered and transmitted fields, we have considered here, for computer simulations, only transmitted fields for the planar configuration and both forward and backward scattering for the cylindrical one, so that there is a spatial frequency low-pass filtering of radius $\sqrt{2} K_{0}$ for the planar case and $2 K_{0}$ for the cylindrical one. According to the theory,
CONTRAST PROFILE
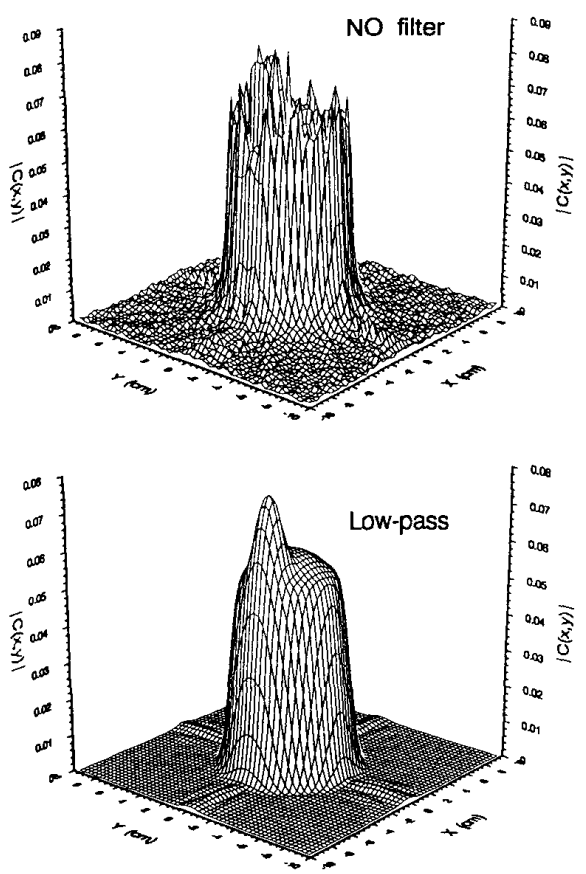

Fig. 20. Low-pass anti-aliasing image filtering on cylindrical systems. The picture shows the originally reconstructed contrast profile and the low-pass filtered one. The image quality is improved by removing high-frequency noise.

the cylindrical algorithm would provide better resolution, sharper corners and fastest rising edges. However, in practice the cylindrical system is not able to measure the backscattered field at elements close to the transmitting one, so that resolution is not as good as theoretically expected and only a slightly better spectral padding is achieved.

Cylindrical and planar geometry algorithms can reconstruct the object in a lossy medium, obtaining a quantitative contrast profile image. Complex permittivity and temperature increment maps can be easily obtained. However, the image quality is distorted by loss correction if backscattered fields are not measured. In that case, a much better image can be obtained without loss correction, at the price of incorrect quantitative values of the contrast profile.

Due to attenuation in the medium, for a given dynamic range of the microwave receiver system, the planar algorithm requires a shorter measurement line than the cylindrical one. However, the larger number of elements in the cylindrical array which measure fields within the specified dynamic range, provide a larger amount of information to be processed, which results in a slightly better image quality.

Mechanical rotation of the object is needed in the planar system in order to take different views, while a synthetic array approach is used in the cylindrical one.

On the other hand, from practical point of view, the planar system could be more effective for imaging an object having a large $y$ dimension, which would not fit inside the circular array. 
In the cylindrical system, the same antennas act as transmitter and receiver elements, so that a microwave switching matrix is needed, with severe isolation requirements between transmitting and receiving elements. The design of the switching matrix is easier if the elements close to the transmitter one do not act as receivers. As these elements measure backscattered fields, some high-frequency components of the image are lost, and the reconstruction is low-pass filtered.

As a global conclusion, although both geometries furnish almost equivalent results, the planar solution is slightly better from the point of view of stability of the reconstructed image, algorithm simplicity and microwave receiver design. The cylindrical geometry takes a slight advantage on the resolution, quantitative image accuracy, and mechanical implementation.

\section{REFERENCES}

[1] R. H. T. Bates, K. L. Garden and T. M. Peters, "Overview of computerized tomography," Proc. IEEE, vol. 71, pp. 356-372, 1983

[2] M. A Stuchly and S.S. Stuchly, "Dielectric properties of biological substances," J. Microwave Power, vol. 15, pp. 19-26, 1980.

[3] L.E. Larsen and J.H. Jacobi, "Microwave offer promise as imaging modality," Diagnostic Imaging, pp. 44-47, Nov. 1982

[4] I. Yamaura, "Measurement of $1.8-2.7 \mathrm{GHz}$ microwave attenuation in the human torso," IEEE Trans. Microwave Theory Technol. pp. 707-710, 1977.

[5] M.F. Adams and A.P. Anderson, "Three-dimensional image-construction technique and its application to coherent microwave diagnostics," Proc. IEEE, vol. 127, pt. H, pp. 138-142, 1980.

[6] C. Süsskind, "Possible use for microwaves in the management of lung disease," Proc. IEEE, vol. 61, pp. 673-674, 1973

[7] L. E. Larsen and J. H. Jacobi, "Microwave scattering parameter imagery of an isolated canine kidney," Med. Phys., vol. 6, pp. 394-403, 1979.

[8] J.H. Jacobi, L.E. Larsen, and C.T. Hast, "Water-immersed microwave antennas and their applications to microwave interrogation of biological target," IEEE Trans. Microwave Theory Technol., vol. 27, pp. 70-77, 1979

[9] A. Broquetas, G. Gastón, M. Hawley, A. Elías, and A. Cardama, "Obtención y medida de dielécticos para construcción de maquetas biológicas en microondas," Actas de la VII Reunión de la Comisión B del Comité Español de la URSI, pp. 449-454, Cuenca, Sept. 1988.

[10] J.C. Lin, "Frequency optimization for microwave imaging of biological tissues," Proc. IEEE, vol. 73, pp. 374-375, 1985.

[11] P.C. Pederson, C.C. Johnson, C.H. Burney, and D. G. Bragg, "An investigation of the use of microwave radiation for pulmonary diagnostics," IEEE Trans. Biomed. Eng., pp. 410-412, 1976.

[12] J.C. Lin and M.I. Clarke, "Microwave imaging of cerebral edemas," Proc. IEEE, vol. 70, pp. 523-525, 1982.

[13] H. Ermet, G. Fülle, and D. Hiller, "Microwave computerized tomography," in 11 th European Microwave Conf., Amsterdam, The Netherlands, 1981, pp. 421-425.

[14] J.C. Bolomey, A. Izadneghadar, L. Jofre, C. Pichot, G. Peronnet, and M. Solaimani, "Microwave diffraction tomography for biomedical applications," IEEE Trans. Microwave Theory Technol., vol. 30, pp. $1998-2000,1982$

[15] M. F. Adams and A.P. Anderson, "Synthetic aperture tomographic (S.A.T.) imaging for microwave diagnostics," IEEE Proc., vol. 129, pt. H, pp. $83-88,1982$.

[16] G. Peronnet et al., "A microwave diffraction tomography system for biomedical applications," in 13th European Microwave Conf., Nuremberg, pp. $529-533,1983$.

[17] J.C. Bolomey, L. Jofre, and G. Peronnet, "On the possible use of microwave active imaging for remote thermal sensing," IEEE Trans. Microwave Theory Technol., vol. 31, pp. 777-781, 1983.

[18] C. Pichot, L. Jofre, G. Peronnet and J.C. Bolomey, "Active microwave imaging of inhomogeneous bodies," IEEE Trans. Atennas. Wave Propagat., vol. 33 , pp. $416-425,1985$.

[19] J.Ch. Bolomey and C. Pichot, "Microwave tomography: From theory to practical imaging systems," Int. J. Imaging Syst. Technol., vol. 1, pp. $119-131$, Dec. 1990.

[20] L. Jofre, M. Hawley, A. Broquetas, E. de los Reyes, M. Ferrando, and A. Elías, "Medical imaging with a micro wave tomographic scanner," IEEE Trans. Biomed. Eng., to be published.

[21] J.M. Rius, M. Ferrando, L Jofre, E. de los Reyes, A. Elías, and A. Broquetas, "Microwave tomography: An algorithm for cylindrical geometries," Electron. Lett., vol. 23, no. 11, pp. 564-565, May 1987.

[22] J. M. Rius, A. Broquetas, M. Ferrando, A Cardama and L. Jofre, "Tomografía de microondas: Reconstrucción de objetos con pérdidas en geometrías cilíndricas," Actas de la VI reunión anual de la comisión B (Campos y Ondas), pp. 511-516, Velencia, Sept. 29-30, 1987

[23] C. Pichot, L. Chommeloux, B. Duchene, N. Joachimowicz, D. Lesselier, W. Tabbara, and J.Ch. Bolomey, "Imagerie microonde et ultrsonore: quelques resultats recents," Actas del III Symposium Naciona del Comité Español de la URSI, pp. 3-5, Gerona, Sept. 22-24, 1986. 